\title{
Density, spin and isospin correlations in low-density two-component Fermi superfluid
}

\author{
A.A. Isayev \\ Kharkov Institute of Physics and Technology, 1 Academicheskaya Str., Kharkov 61108, Ukraine \\ J. Yang \\ Dept. of Physics and Center for Space Science and Technology, Ewha Womans University, Seoul 120-750, Korea \\ E-mail: jyang@ewha.ac.kr
}

Received December 6, 2005, revised February 23, 2006

\begin{abstract}
Finding the distinct features of the crossover from the regime of large overlapping Cooper pairs to the limit of non-overlapping pairs of fermions (Shafroth pairs) in multi-component Fermi systems remains a topical problem in a quantum many-body theory. Here this transition is studied by calculating the two-body density, spin and isospin correlation functions in dilute two-component Fermi superfluid, taking as an example an infinite system of protons and neutrons (nuclear matter). It is shown that criterion of the crossover (Phys. Rev. Lett. 95, 090402 (2005)), formulated for ultracold fermionic atomic gases and consisting in the change of the sign of the density correlation function at low momentum transfer, fails to describe correctly the density-driven BEC-BCS transition at finite isospin asymmetry or finite temperature. As an unambiguous signature of the BEC-BCS transition, one can use the presence (BCS regime) or absence (BEC regime) of the singularity in the momentum distribution of the quasiparticle density of states.
\end{abstract}

PACS: 21.65.+f, 21.30.Fe, 71.10.Ay

Keywords: BCS-BEC transition, bound state, correlation function, quasiparticle density of states.

\section{Introduction}

The transition from BCS superconductivity to Bose-Einstein condensation (BEC) occurs in a Fermi system if either the density is decreased or the attractive interaction between fermions is increased sufficiently. The crossover from large overlapping Cooper pairs to tightly bound pairs of fermions can be described on the basis of BCS theory if the effects of fluctuations are disregarded. This transition has been studied in excitonic semiconductors [1], ordinary superconductors [2], in an attractive Fermi gas [3], nuclear [4,5] and quark [6] matter. Although the BCS and BEC limits are physically quite different, the crossover between them was found to be smooth within the BCS theory.

The recent upsurge of interest in the BEC-BCS crossover was caused by finding BCS pairing in ultracold quantum atomic gases [7-9]. Ultracold atomic gases provide an experimental playground for testing pairing phenomena due to the possibility of controling the inter-atomic interactions via a magnetically-tuned Feshbach resonance. In low-density systems of two types of fermions, such as ultracold gases of alkali atoms, the interaction can be characterized by a single parameter, the $s$-wave scattering length $a_{s}$. If the scattering length is negative, one expects fermions in the two hyperfine levels to pair. On the opposite side from the position of the Feshbach resonance, when the scattering length is positive, pairs of atoms can form weakly bound states in the form of molecules. In this regime, one expects the system to consist of a BEC of these molecules. In fact, as one goes through the resonance starting from the negative scattering lengths, the Cooper pairs in the BCS state shrink in size and go over continuously into a Bose - Einstein condensate of bound states. An important question arises what are the observable features of the BCS-BEC transition.

Such signatures were attempted to be found in a recent study [10]. The authors of that study argued that 
the density correlation function of a two-component ultracold fermionic gas of atoms changes sign at low-momentum transfer and this represents an unambiguous signature of the BEC-BCS crossover. Here we would like to extend their calculations taking into account additional factors: finite isospin asymmetry (nonequal densities of fermions of different species) or finite temperature. Both factors are missing in the study of Ref. 10. As an example of two-component Fermi system, we choose nuclear matter, i.e., an infinite Fermi system consisting of neutrons and protons. In free space the strongest internucleon interaction is the interaction between a neutron and a proton in the state with the total spin of a pair $S=1$, leading to the formation of a real bound state - deuteron. At not too low densities, a condensate of neutron - proton (np) Cooper pairs is formed in nuclear matter [11,12]. Under decreasing density, np Cooper pairs go over to BEC of deuterons. During this transition the chemical potential changes sign at a certain critical density (Mott transition), approaching half of the deuteron binding energy at ultralow densities [13,14]. We will study density, spin and isospin correlations in low-density nuclear matter and will show that the criterion of Ref. 10 fails to provide a correct description of the density-driven BEC-BCS crossover and cannot serve as the universal feature of transition between two states of a Fermi system.

\section{Basic equations}

The states of two-component Fermi superfluid are described by the normal $f$ and anomalous $g$ distribution functions of fermions

$$
f_{\kappa_{1} \kappa_{2}}=\operatorname{Tr} \varrho a_{\kappa_{2}}^{+} a_{\kappa_{1}}, g_{\kappa_{1} \kappa_{2}}=\operatorname{Tr} \varrho a_{\kappa_{2}} a_{\kappa_{1}},
$$

where $\kappa \equiv(\mathbf{k}, \sigma, \tau), \mathbf{k}$ is the momentum, $\sigma(\tau)$ is the projection of spin (isospin) on the third axis, and $\varrho$ is the density matrix of the system. For nuclear matter, $\tau=+1 / 2$ corresponds to neutrons and $\tau=-1 / 2$ corresponds to protons. We shall study np pairing correlations in the pairing channel with total spin $S$ and isospin $T$ of a pair $S=1, T=0$ and the projections $S_{z}=T_{z}=0$. In this case the distribution functions for isospin asymmetric nuclear matter have the structure

$$
\begin{aligned}
& f(\mathbf{k})=f_{00}(\mathbf{k}) \sigma_{0} \tau_{0}+f_{03}(\mathbf{k}) \sigma_{0} \tau_{3}, \\
& g(\mathbf{k})=g_{30}(\mathbf{k}) \sigma_{3} \sigma_{2} \tau_{2},
\end{aligned}
$$

where $\sigma_{i}$ and $\tau_{k}$ are the Pauli matrices in spin and isospin spaces, respectively. Using the minimum principle of the thermodynamic potential and the procedure of block diagonalization [12], one can obtain expressions for the distribution functions

$$
\begin{aligned}
& f_{00}(\mathbf{k})=\frac{1}{2}-\frac{\xi_{k}}{4 E_{k}}\left(\tanh \frac{E_{k}^{+}}{2 T}+\tanh \frac{E_{k}^{-}}{2 T}\right) \\
& f_{03}(\mathbf{k})=\frac{1}{4}\left(\tanh \frac{E_{k}^{+}}{2 T}-\tanh \frac{E_{k}^{-}}{2 T}\right) \\
& g_{30}(\mathbf{k})=-\frac{\Delta(\mathbf{k})}{4 E_{k}}\left(\tanh \frac{E_{k}^{+}}{2 T}+\tanh \frac{E_{k}^{-}}{2 T}\right)
\end{aligned}
$$

Here

$$
E_{k}^{ \pm}=E_{k} \pm \delta \mu=\sqrt{\xi_{k}^{2}+\Delta^{2}(\mathbf{k})} \pm \delta \mu, \quad \xi_{k}=\frac{\mathbf{k}^{2}}{2 m}-\mu,
$$

$\Delta$ being the energy gap in the quasiparticle excitation spectrum, $m$ being the effective nucleon mass, $\mu$ and $\delta \mu$ being half of the sum and half of the difference of the neutron and proton chemical potentials, respectively.

Equations governing np pairing correlations in the $S=1, T=0$ pairing channel can be obtained on the base of the variational approach for the thermodynamic potential $[14,15]$ and have the form

$$
\begin{aligned}
& \Delta(\mathbf{k})=-\frac{1}{V} \sum_{\mathbf{k}^{\prime}} V\left(\mathbf{k}, \mathbf{k}^{\prime}\right) \frac{\Delta\left(\mathbf{k}^{\prime}\right)}{2 E_{k^{\prime}}}\left(1-f\left(E_{k^{\prime}}^{+}\right)-f\left(E_{k^{\prime}}^{-}\right)\right), \\
& \varrho=\frac{2}{V} \sum_{\mathbf{k}}\left(1-\frac{\xi_{k}}{E_{k}}\left[1-f\left(E_{k}^{+}\right)-f\left(E_{k}^{-}\right)\right]\right) \equiv \frac{2}{V} \sum_{\mathbf{k}} n_{k}, \\
& \alpha \varrho=\frac{2}{V} \sum_{\mathbf{k}}\left(f\left(E_{k}^{-}\right)-f\left(E_{k}^{+}\right)\right),
\end{aligned}
$$

where $f(E)$ is the Fermi distribution function. Equation (7) is the equation for the energy gap $\Delta$ and Eqs. (8), (9) are equations for the total density $\varrho=\varrho_{p}+\varrho_{n}$ and neutron excess $\delta \varrho=\varrho_{n}-\varrho_{p} \equiv \alpha \varrho(\alpha$ being the asymmetry parameter). Note that since we are considering a unitary superfluid state $\left(\Delta \Delta^{+} \propto I\right)$, Eqs. (7)-(9) formally coincide with the equations for two-component isospin asymmetric superfluid with singlet spin pairing between unlike fermions. Introducing the anomalous density

$$
\psi(\mathbf{k})=<a_{n, k}^{+} a_{p,-k}^{+}>=\frac{\Delta(\mathbf{k})}{2 E_{k}}\left[1-f\left(E_{k}^{+}\right)-f\left(E_{k}^{-}\right)\right]
$$

and using Eq. (8), one can represent Eq. (7) for the energy gap in the form

$$
\frac{k^{2}}{m} \psi(\mathbf{k})+\left(1-n_{k}\right) \sum_{\mathbf{k}^{\prime}} V\left(\mathbf{k}, \mathbf{k}^{\prime}\right) \psi\left(\mathbf{k}^{\prime}\right)=2 \mu \psi(\mathbf{k}) .
$$

In the limit of vanishing density, $n_{k} \rightarrow 0$, Eq. (10) goes over into the Schrödinger equation for the deuteron bound state $[5,13]$. The corresponding energy 
eigenvalue is equal to $2 \mu$. The change in the sign of the mean chemical potential $\mu$ of neutrons and protons under decreasing density of nuclear matter signals the transition from the regime of large overlapping np Cooper pairs to the regime of non-overlapping bound states (deuterons).

Let us consider the two-body density correlation function

$$
\begin{aligned}
& \mathcal{D}\left(\mathbf{x}, \mathbf{x}^{\prime}\right)=\operatorname{Tr} \varrho \Delta \hat{n}(\mathbf{x}) \Delta \hat{n}\left(\mathbf{x}^{\prime}\right), \Delta \hat{n}(\mathbf{x})=\hat{n}(\mathbf{x})-\hat{n}, \\
& \hat{n}(\mathbf{x}) \equiv \sum_{\sigma \tau} \Psi_{\sigma \tau}^{+}(\mathbf{x}) \psi_{\sigma \tau}(\mathbf{x})= \\
& =\frac{1}{V} \sum_{\sigma \tau \mathbf{k} \mathbf{k}^{\prime}} \exp \left(i\left(\mathbf{k}^{\prime}-\mathbf{k}\right) \mathbf{x}\right) a_{\mathbf{k} \sigma \tau}^{+} a_{\mathbf{k}^{\prime} \sigma \tau}, \\
& \hat{n}=\frac{1}{V} \sum_{\sigma \tau \mathbf{k}} a_{\mathbf{k} \sigma \tau}^{+} a_{\mathbf{k} \sigma \tau} .
\end{aligned}
$$

Its general structure in the spatially uniform and isotropic case reads [16]

$$
\mathcal{D}\left(\mathbf{x}, \mathbf{x}^{\prime}\right)=\varrho \delta(\mathbf{r})+\varrho D(r), \mathbf{r}=\mathbf{x}-\mathbf{x}^{\prime} .
$$

The function $D(r)$ is called the density correlation function as well. We will be just interested in the behavior of the function $D(r)$. The trace in Eq. (10) can be calculated, using definitions (1) and Wick rules. Taking into account Eqs. (2) and going to the Fourier representation

$$
D(q)=\int d^{3} \mathbf{r} \exp (i \mathbf{q r}) D(r),
$$

one can get

$$
D(q)=I_{g}^{30}(q)-I_{f}^{00}(q)-I_{f}^{03}(q)
$$

where

$$
\begin{aligned}
& I_{f}^{00}(q)=\frac{4}{\pi^{3} \varrho} \int_{0}^{\infty} d r r^{2} j_{0}(r q)\left[\int_{0}^{\infty} d k k^{2} f_{00}(k) j_{0}(r k)\right]^{2}, \\
& I_{f}^{03}(q)=\frac{4}{\pi^{3} \varrho} \int_{0}^{\infty} d r r^{2} j_{0}(r q)\left[\int_{0}^{\infty} d k k^{2} f_{03}(k) j_{0}(r k)\right]^{2}, \\
& I_{g}^{30}(q)=\frac{4}{\pi^{3} \varrho} \int_{0}^{\infty} d r r^{2} j_{0}(r q)\left[\int_{0}^{\infty} d k k^{2} g_{30}(k) j_{0}(r k)\right]^{2} .
\end{aligned}
$$

Here $j_{0}$ is the spherical Bessel function of the first kind and zeroth order. Functions $I_{f}^{00}, I_{f}^{03}$, and $I_{g}^{30}$ represent the normal and anomalous contributions to the density correlation function. Analogously, we can consider the two-body spin correlation function

$$
S_{\mu \nu}\left(\mathbf{x}, \mathbf{x}^{\prime}\right)=\operatorname{Tr} \varrho \Delta \hat{S}_{\mu}(\mathbf{x}) \Delta \hat{S}_{v}\left(\mathbf{x}^{\prime}\right),
$$

$$
\begin{gathered}
\Delta \hat{s}_{\mu}(\mathbf{x})=\hat{s}_{\mu}(\mathbf{x})-\hat{s}_{\mu}, \\
\hat{s}_{\mu}(\mathbf{x}) \equiv \frac{1}{2} \sum_{\sigma \sigma^{\prime} \tau} \psi_{\sigma \tau}^{+}(\mathbf{x})\left(\sigma_{\mu}\right)_{\sigma \sigma^{\prime}} \Psi_{\sigma^{\prime} \tau}(\mathbf{x})= \\
=\frac{1}{2 V} \sum_{\sigma \sigma^{\prime} \tau \mathbf{k} \mathbf{k}^{\prime}} \exp \left(i\left(\mathbf{k}^{\prime}-\mathbf{k}\right) \mathbf{x}\right) a_{\mathbf{k} \sigma \tau}^{+}\left(\sigma_{\mu}\right)_{\sigma \sigma^{\prime}} a_{\mathbf{k}^{\prime} \sigma^{\prime} \tau} \\
\hat{s}_{\mu}=\frac{1}{2 V} \sum_{\sigma \sigma^{\prime} \tau \mathbf{k}} a_{\mathbf{k} \sigma \tau}^{+}\left(\sigma_{\mu}\right)_{\sigma \sigma^{\prime}} a_{\mathbf{k} \sigma^{\prime} \tau}
\end{gathered}
$$

and the two-body isospin correlation function

$$
\begin{aligned}
& \mathcal{T}_{\mu \nu}\left(\mathbf{x}, \mathbf{x}^{\prime}\right)=\operatorname{Tr} \varrho \Delta \hat{t}_{\mu}(\mathbf{x}) \Delta \hat{t}_{v}\left(\mathbf{x}^{\prime}\right), \\
& \Delta \hat{t}_{\mu}(\mathbf{x})=\hat{t}_{\mu}(\mathbf{x})-\hat{t}_{\mu}, \\
& \hat{t}_{\mu}(\mathbf{x}) \equiv \frac{1}{2} \sum_{\sigma \tau \tau^{\prime}} \Psi_{\sigma \tau}^{+}(\mathbf{x})\left(\tau_{\mu}\right)_{\tau \tau^{\prime}} \Psi_{\sigma \tau^{\prime}}(\mathbf{x})= \\
& =\frac{1}{2 V} \sum_{\sigma \tau \tau^{\prime} \mathbf{k} \mathbf{k}^{\prime}} \exp \left(i\left(\mathbf{k}^{\prime}-\mathbf{k}\right) \mathbf{x}\right) a_{\mathbf{k} \sigma \tau}^{+}\left(\tau_{\mu}\right)_{\tau \tau^{\prime}} a_{\mathbf{k}^{\prime} \sigma \tau^{\prime}}, \\
& \hat{t}_{\mu}=\frac{1}{2 V} \sum_{\sigma \tau \tau^{\prime} \mathbf{k}} a_{\mathbf{k} \sigma \tau}^{+}\left(\tau_{\mu}\right)_{\tau \tau^{\prime}} a_{\mathbf{k} \sigma \tau^{\prime}} .
\end{aligned}
$$

Their general structure for isospin asymmetric nuclear matter without spin polarization is

$$
\begin{aligned}
& S_{\mu \nu}\left(\mathbf{x}, \mathbf{x}^{\prime}\right)=\frac{\varrho}{4} \delta_{\mu \nu} \delta(\mathbf{r})+\varrho S_{\mu \nu}(r), \\
& \mathcal{T}_{\mu \nu}\left(\mathbf{x}, \mathbf{x}^{\prime}\right)=\frac{\varrho}{4} \delta_{\mu \nu} \delta(\mathbf{r})+\frac{\alpha \varrho}{4} i \varepsilon_{\mu \nu 3} \delta(\mathbf{r})+\varrho T_{\mu \nu}(r) .
\end{aligned}
$$

Then, calculating traces in Eqs. (14), (15), for the Fourier transforms of the spin and isospin correlation functions, one can get

$$
\begin{aligned}
S_{\mu \nu}(q)= & -\frac{1}{4}\left\{\delta_{\mu \nu}\left(I_{f}^{00}(q)+I_{f}^{03}(q)\right)+\right. \\
& \left.+\left(\delta_{\mu \nu}-2 \delta_{3 \mu} \delta_{3 v}\right) I_{g}^{30}(q)\right\}, \\
T_{\mu \nu}(q)= & -\frac{1}{4}\left\{\delta_{\mu \nu}\left(I_{f}^{00}(q)+I_{g}^{30}(q)\right)-\right. \\
& \left.-\left(\delta_{\mu \nu}-2 \delta_{3 \mu} \delta_{3 v}\right) I_{f}^{03}(q)\right\} .
\end{aligned}
$$

Note that if one puts $v=\mu=3$ in Eqs. (18), (19), one gets the longitudinal spin $S^{l}$ and isospin $T^{l}$ correlation functions, while setting $\mu, v=1,2$ gives the transverse spin and isospin correlation functions

$$
\begin{array}{r}
S_{\mu \nu}^{t}(q)=-\frac{\delta_{\mu \nu}}{4}\left(I_{f}^{00}(q)+I_{f}^{03}(q)+I_{g}^{30}(q)\right) \equiv \delta_{\mu \nu} S^{t}(q), \\
\mu, v=1,2, \\
T_{\mu \nu}^{t}(q)=-\frac{\delta_{\mu \nu}}{4}\left(I_{f}^{00}(q)-I_{f}^{03}(q)+I_{g}^{30}(q)\right) \equiv \delta_{\mu \nu} T^{t}(q) .
\end{array}
$$


The following relationships between the correlation functions hold true:

$$
S^{l}(q)=\frac{D(q)}{4}, S^{t}(q)=T^{l}(q) .
$$

At zero temperature and zero momentum transfer, the correlation functions satisfy the sum rule

$$
\begin{aligned}
& S^{t}(q=0)=T^{l}(q=0)= \\
& =-\frac{1}{2 \pi^{2} \varrho} \int d k k^{2}\left(f_{00}^{2}(k)+f_{03}^{2}(k)+g_{30}^{2}(k)\right)=-\frac{1}{4},
\end{aligned}
$$

where the right-hand side is independent of density and isospin asymmetry. Besides, the transverse isospin correlation function satisfies the relationship

$$
\begin{aligned}
T^{t}(q=0) & =-\frac{1}{2 \pi^{2} \varrho} \int d k k^{2}\left(f_{00}^{2}(k)-f_{03}^{2}(k)+g_{30}^{2}(k)\right)= \\
& =-\frac{1-\alpha}{4}
\end{aligned}
$$

where the right-hand side is independent of density.

\section{Correlation functions in dilute two-component Fermi superfluid}

Further for numerical calculations we shall use the effective zero range force developed in Ref. 17 to reproduce the pairing gap in the $S=1, T=0$ pairing channel with the Paris nucleon potential:

$$
V\left(\mathbf{r}_{1}, \mathbf{r}_{2}\right)=v_{0}\left\{1-\eta\left[\frac{\varrho\left(\frac{\mathbf{r}_{1}+\mathbf{r}_{2}}{2}\right)}{\varrho_{0}}\right]^{\gamma}\right\} \delta\left(\mathbf{r}_{1}-\mathbf{r}_{2}\right)
$$

where $\varrho_{0}=0.16 \mathrm{fm}^{-3}$ is the nuclear saturation density, $v_{0}=-530 \mathrm{MeV} \cdot \mathrm{fm}^{3}, \eta=0, m=m_{G}, m_{G}$ being the effective mass, corresponding to the Gogny force D1S [18]. Besides, in the gap equation (7), Eq. (24) must be supplemented with a cutoff parameter, $\varepsilon_{c}=60 \mathrm{MeV}$.

To find the correlation functions, first, one should solve the gap Eq. (7) self-consistently with Eqs. (8), (9). Then the correlation functions can be determined directly from Eqs. (13), (18), and (19). The results of numerical determination of the energy gap as a function of density for different asymmetries at zero temperature are shown in Fig. 1. As one can see, with increasing asymmetry the magnitude of the energy gap is decreased and the density interval, where a np condensate exists, shrinks to lower density. In reality solutions exist for any $\alpha<1$ (the phase curves for larger values of $\alpha$ are not shown in Fig. 1) and correspond to

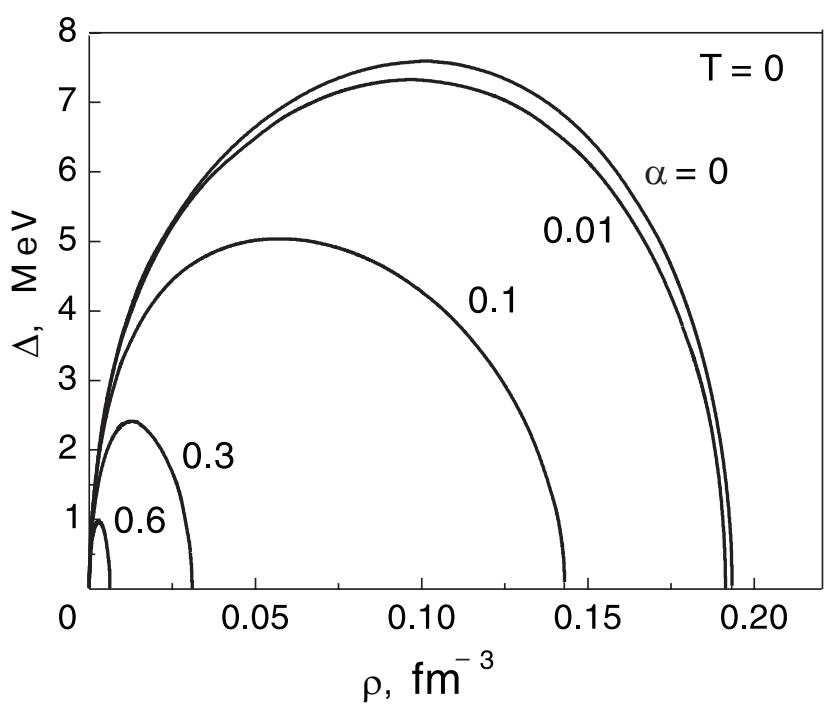

Fig. 1. Energy gap as a function of density at zero temperature and different asymmetries.

the formation of BEC of deuterons at very low densities of nuclear matter.

\section{Correlation functions for isospin symmetric case and zero temperature}

Now we consider the correlation functions $D(q)$ and $S^{t}(q)$ for symmetric nuclear matter at zero temperature, depicted in Fig. 2 (at $\left.\alpha=0, T^{t}(q)=S^{t}(q)\right)$. The density correlation function changes sign at low momentum transfer when the system smoothly evolves

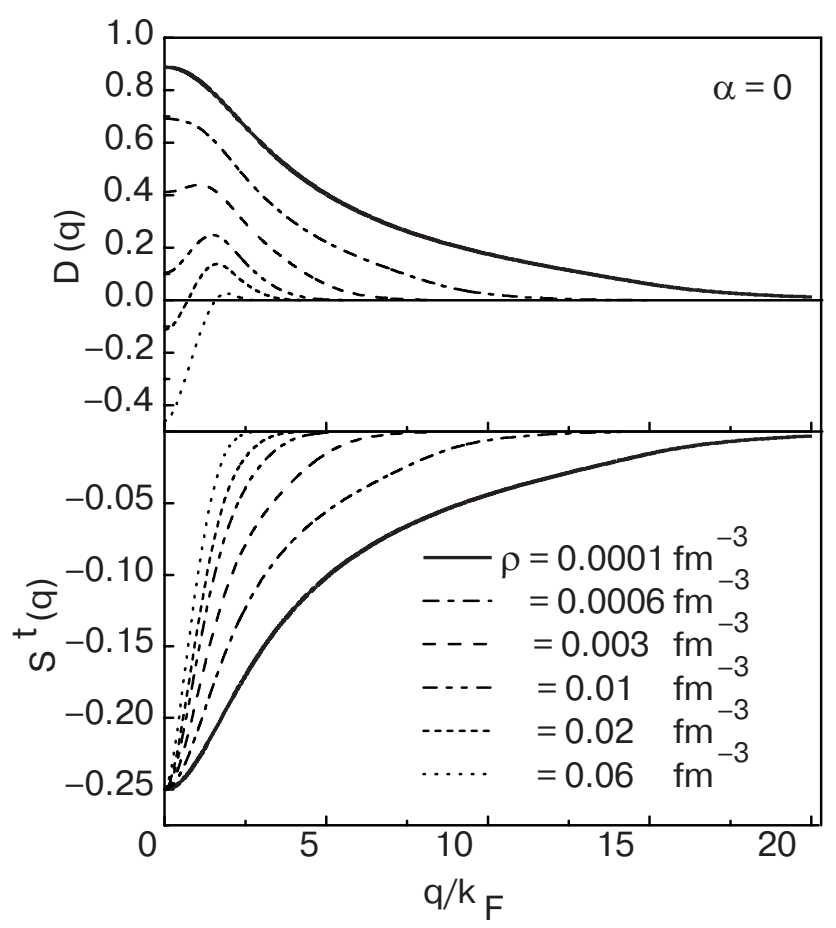

Fig. 2. Density and transverse spin correlation functions as functions of momentum at zero temperature and different densities for symmetric nuclear matter. 
from the BEC regime to the BCS one. These two regimes are distinguished by the negative and positive values of the chemical potential $\mu$, respectively. In view of Eq. (21), the longitudinal spin correlation function $S^{l}(q)$ changes the sign through the $\mathrm{BEC}-\mathrm{BCS}$ crossover as well. The transverse spin correlation function, and, according to Eq. (21), the longitudinal and transverse isospin correlation functions change fluently between BEC and BCS limits. The behavior of the density correlation function in isospin symmetric case at zero temperature agrees qualitatively with the behavior of the density correlation function in ultracold fermionic atom gas with singlet pairing of fermions [10]. In Ref. 10, the change in the sign of the density correlation function at low momentum transfer was considered as a signature of the BEC-BCS crossover. We will extend their calculations with account of finite isospin asymmetry and finite temperature.

Figure 3 (top) shows the dependence of the density correlation function $D(q=0)$ at zero momentum transfer from density for symmetric nuclear matter. The density correlation function changes sign between BEC and BCS limits, that is also true for the longitudinal spin correlation function $S^{l}(q=0)$. Corresponding normal $I_{f}^{00}(q=0)$ and anomalous $I_{g}^{30}(q=0)$ contributions to $D(q=0)$ are shown in Fig. 3 (bottom). According to Eq. (20) and the sum rule for

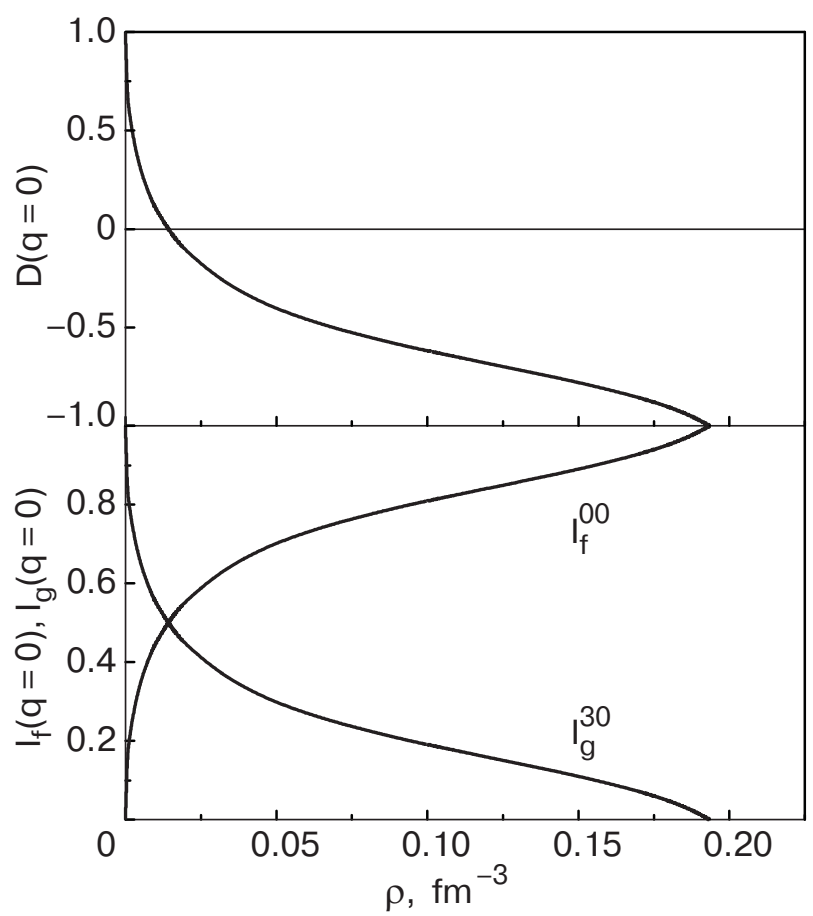

Fig. 3. Zero-momentum transfer density correlation function $D(q=0)$, and its normal $I_{f}^{00}(q=0)$ and anomalous $I_{g}^{30}(q=0)$ contributions as functions of density at zero temperature for symmetric nuclear matter.
$S^{t}(q=0)$, in isospin symmetric case they satisfy the relationship $I_{f}^{00}(0)+I_{g}^{30}(0)=1$.

\section{Correlation functions for isospin asymmetric case and zero temperature}

Now we will present the results of numerical determination of the correlation functions at finite isospin asymmetry and zero temperature. The behavior of the density correlation function $D(q)$ as a function of momentum at zero temperature for different asymmetries and densities is shown in Fig. 4. In accordance with the above criterion, the transition density from BEC to BCS state shifts to lower densities with increasing asymmetry (at $\alpha=0.6$ and $\varrho=0.01 \mathrm{fm}^{-3}$ we have already the normal state, and, consequently, only three curves are shown in the bottom right part of Fig. 4). At strong enough isospin asymmetry (e.g., $\alpha \gtrsim 0.6$ ) we would have only the BCS state. It is clearly seen from Fig. 5, where the density correlation function $D(q=0)$ at zero momentum transfer is shown as a function of density for a set of various isospin asymmetry parameters and zero temperature. For large isospin asymmetry the function $D(q=0)$ is always negative, that would correspond to the BCS state for all densities where a np condensate exists. Obviously, this conclusion is contradicted by the behavior of the mean chemical potential $\mu$, being negative at very low densities for any $\alpha<1$, and, hence, giving evidence for the formation of a BEC of bound states [14]. Thus, at strong isospin asymmetry the criterion of the crossover, based on the change of the sign of the density correlation function, fails to predict the transition to

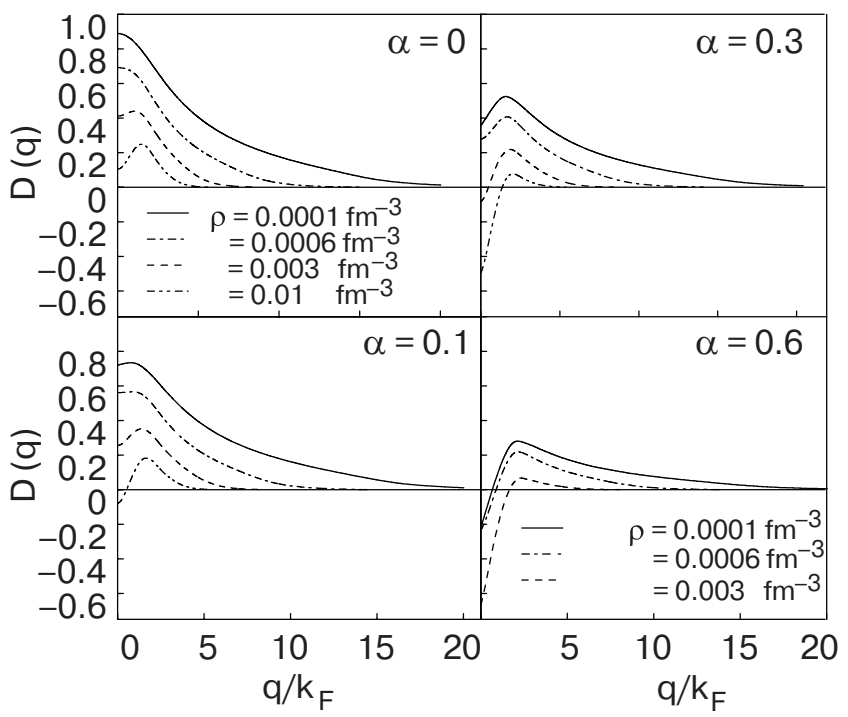

Fig. 4. Density correlation function $D(q)$ as a function of momentum at zero temperature for different asymmetries and densities. 


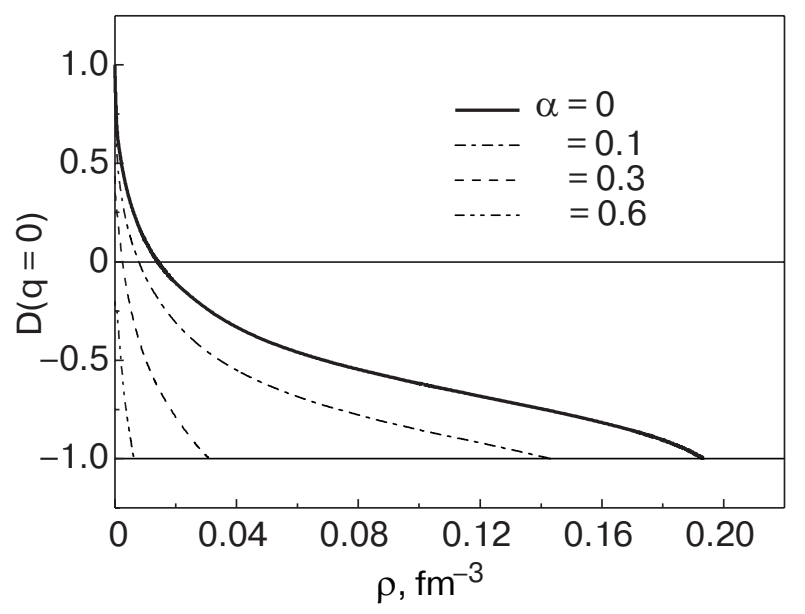

Fig. 5. Density correlation function $D(q=0)$ as a function of density at zero temperature for different isospin asymmetry parameters.

the BEC of bound states in a low-density two-component Fermi system.

Figures 6 and 7 show the transverse spin and transverse isospin correlation functions as functions of momentum at zero temperature for different asymmetries and densities. These correlation functions at zero temperature satisfy the sum rules (22), (23) and change smoothly between their limiting values $S^{t}(q=0)$, $T^{t}(q=0)$, and zero value, respectively.

\section{Density correlation function for isospin symmetric case and nonzero temperature}

Now we consider symmetric nuclear matter at finite temperature. Figure 8 shows the dependence of the density correlation function $D(q=0)$ at zero momentum transfer as a function of density for a set of vari-

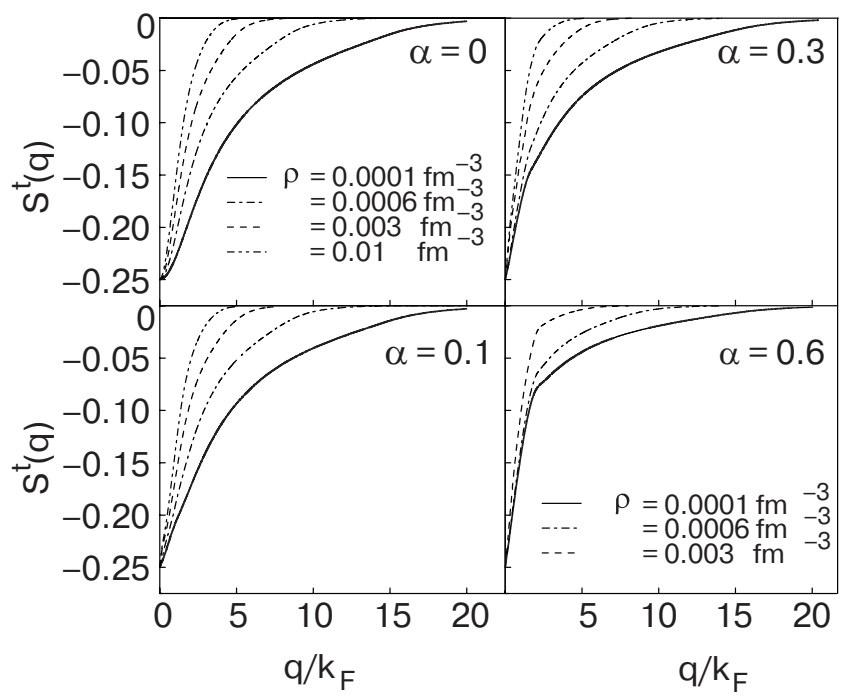

Fig. 6. Same as in Fig. 4, but for the transverse spin correlation function $S^{t}(q)$.

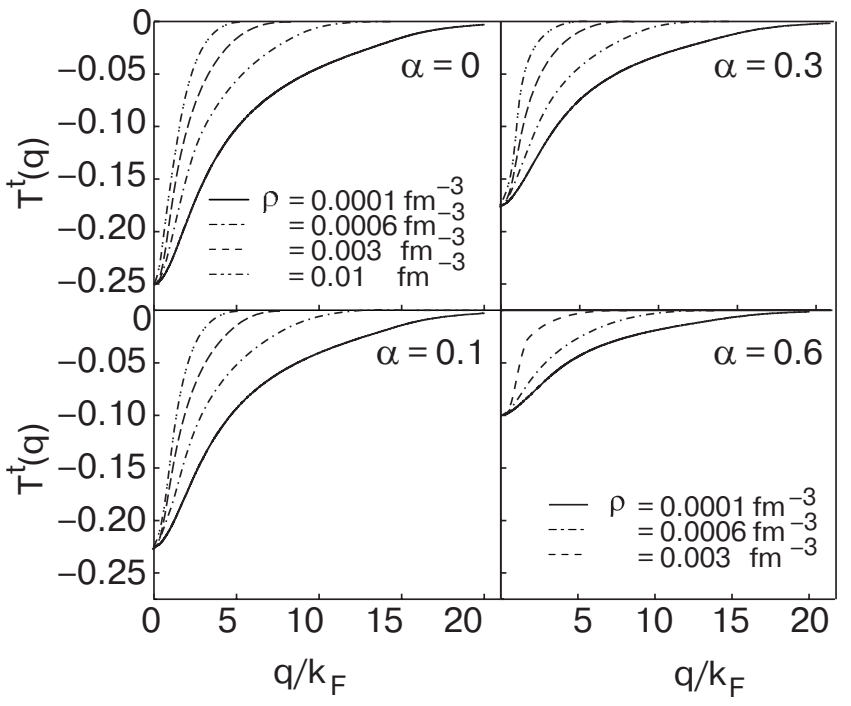

Fig. 7. Same as in Fig. 4, but for the transverse isospin correlation function $T^{t}(q)$.

ous temperatures. It is seen that for not too high temperatures the density correlation function is non-monotone and twice changes sign in the region of low densities. Hence, in accordance with the above criterion, we would have the density interval $\varrho_{1}<\varrho<\varrho_{2}$ with the BEC state, surrounded by the density regions with the BCS state. However, this conclusion is contradicted by the behavior of the mean chemical potential $\mu$ for these temperatures, being a monotone function of density and giving indication of the formation of the BEC state at low densities $(\mu<0)$ and the BCS state at larger densities $(\mu>0)$. Thus, at finite temperature the criterion of the crossover, formulated in Ref. 10, fails to provide the correct description of the transition between two regimes.

Qualitatively the boundary between BEC and BCS states corresponds to the point where the chemical po-

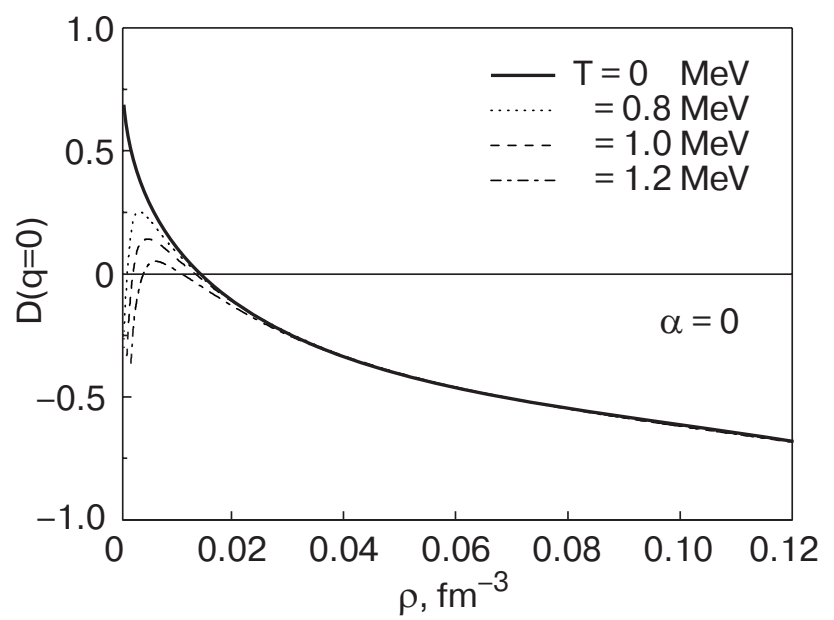

Fig. 8. Density correlation function $D(\mathbf{q}=0)$ as a function of density at different temperatures for symmetric nuclear matter. 
tential changes sign. According to Eq. (6), at this point there is a qualitative change in the quasiparticle excitation spectrum: the momentum corresponding to the minimum in the excitation spectrum shifts from a finite value in the BCS state to the zero value in the BEC state. The appearance of the minimum in the quasiparticle excitation spectrum is clearly reflected in the quasiparticle density of states

$$
v(k)=\frac{1}{\pi^{2}} \frac{k^{2}}{\left|d E_{k} / d k\right|} .
$$

The density of states $v(k)$ is singular in the stationary points of the excitation spectrum $\left(d E_{k} / d k=0\right)$, except the point $k=0$, where $v(k)$ vanishes, assuming that $d E_{k} / d k$ goes to zero no faster than $k^{2}$. Thus, the appearance of the singularity in the momentum distribution of the quasiparticle density of states represents the universal signature of the BEC-BCS transition (see also Ref. 19).

\section{Discussion and Conclusions}

The transition from large overlapping Cooper pairs to tightly bound pairs of fermions can be described entirely within the mean-field approximation (BCS theory). Indeed, in free-space limit the gap equation reduces to the Schrödinger equation for bound pairs. An important question appears: to what extent can the BCS theory be trusted and what is the role of the beyond-mean-field effects? In order to give a correct answer, it is necessary to compare calculations based on the BCS theory with the results of more exact schemes. The value of $\mu / \varepsilon_{F}$ for $\eta \equiv\left(a_{S} k_{F}\right)^{-1}=0$ is usually referred to as the «unitary limit» [20]. In this limit, when the density is held fixed, one expects that all sensitivity to the detailed features of the interaction is lost. As a result, this limit is particularly sensitive to many-body correlation effects, and the BCS calculations, based on the single-parameter potential, predict this value as being equal to 0.59 in the isospin symmetric case and at zero temperature [19]. Recent, more-accurate calculations based on the Green's function Monte Carlo approach [21] have lowered the upper limit on this ratio to $(0.44 \pm 0.01)$, which shows that beyond-mean-field effects account for at least a $25 \%$ improvement in the binding energy over the mean-field result. Thus, the consideration based on the BCS theory gives a qualitatively correct picture of the BEC-BCS transition, but further work is needed in order to include many-body effects in a quantitative way and to study the changes in the features of the BEC-BCS crossover.

In summary, we have calculated the density, spin and isospin correlation functions in low-density two-component Fermi superfluid, taking as an example superfluid nuclear matter with a neutron - proton condensate. It is shown that the transverse spin, and longitudinal and transverse isospin correlation functions satisfy the sum rule at zero momentum transfer and zero temperature. In Ref. 10 it was learned that the density correlation function in two-component ultracold fermionic atom gas with singlet pairing of fermions changes sign at low momentum transfer across the BEC-BCS transition, driven by changing the scattering length of the interaction at zero temperature. We have shown that for spin triplet pairing the longitudinal spin correlation function plays an analogous role to the density correlation function and changes the sign at low momentum transfer across the crossover in symmetric nuclear matter at zero temperature. However, while giving the satisfactory description of the density-driven BEC-BCS crossover in dilute nuclear matter at zero temperature for isospin symmetric case, this criterion fails to provide the correct description of the crossover at finite isospin asymmetry (nonequal densities of fermions of different species) or finite temperature. Hence, the criterion in Ref. 10 cannot be considered as the universal indication of the BEC-BCS transition. During the Mott transition, when the chemical potential changes sign, there is a qualitative change in the quasiparticle energy spectrum: the minimum shifts from a finite (BCS state) to zero-momentum value (BEC state). As such, the presence (BCS) or absence (BEC) of the singularity in the momentum distribution of the quasiparticle density of states represents the universal signature of the BEC-BCS transition.

From the general point of view, we have studied the possible features of the BEC-BCS crossover in a low-density two-component Fermi superfluid within the mean-field approach. The conclusions of this study hold true not only for nuclear matter, taken here as an example, but, for instance, for a two-component ultracold fermionic gas of atoms (e.g., $\left.{ }^{6} \mathrm{Li},{ }^{40} \mathrm{~K}\right)$. In the last case, the presence or absence of the singularity in the quasiparticle density of states can be determined spectroscopically [22].

J.Y. acknowledges support from the CHEP SRC program of KOSEF(Korea).

1. D.M. Eagles, Phys. Rev. 186, 456 (1969); L.V. Keldysh and A.N. Kozlov, Sov. Phys. JETP 27, 521 (1968).

2. A.J. Leggett, J. Phys. (Paris) 41, C7 (1980).

3. P. Nozieres and S. Schmitt-Rink, J. Low Temp. Phys. 59, 195 (1985).

4. T. Alm, B.L. Friman, G. Röpke, et al., Nucl. Phys. A551, 45 (1993). 
5. M. Baldo, U. Lombardo, and P. Schuck, Phys. Rev. C52, 975 (1995).

6. T. Sch!afer and F. Wilczek, Phys. Rev. Lett. 82, 3956 (1999).

7. C.A. Regal, M. Greiner, and D.S. Jin, Phys. Rev. Lett. 92, 040403 (2004).

8. J. Kinast, S.L. Hemmer, M.E. Gehm, A. Turlapov, and J.E. Thomas, Phys. Rev. Lett. 92, 150402 (2004).

9. M.W. Zwierlein, C.A. Stan, C.H. Schunck, S.M.F. Raupach, A.J. Kerman, and W. Ketterle, Phys. Rev. Lett. 92, 120403 (2004).

10. B. Mihaila, S. Gaudio, K.B. Blagoev, A.V. Balatsky, P.B. Littlewood, and D.L. Smith, Phys. Rev. Lett. 95, 090402 (2005).

11. A. Sedrakian and U. Lombardo, Phys. Rev. Lett. 84, $602(2000)$.

12. A.I. Akhiezer, A.A. Isayev, S.V. Peletminsky, et al., Phys. Rev. C63, 021304(R) (2001).

13. U. Lombardo, P. Nozieres, P. Schuck, et al., Phys. Rev. C64, 064314 (2001).
14. A.A. Isayev, S.I. Bastrukov, and J. Yang, Nucl. Phys. A734, E112 (2004); Phys. Atom. Nucl. 67, 1840 (2004).

15. A.I. Akhiezer, A.A. Isayev, S.V. Peletminsky, and A.A. Yatsenko, Fiz. Nizk. Temp. 24, 75 (1998) [Low Temp. Phys. 2456 (1998)].

16. L.D. Landau and E.M. Lifshitz, Statistical Physics, Part 1, Pergamon Press (1980).

17. E. Garrido, P. Sarriguren, E. Moya de Guerra, et al., Phys. Rev. C63, 037304 (2001).

18. J.F. Berger, M. Girod and D. Gogny, Comp. Phys. Comm. 63, 365 (1991).

19. M. Parish, B. Mihaila, E.M. Timmermans, K.B. Blagoev, and P.B. Littlewood, Phys. Rev. B71, 064513 (2005).

20. H. Heiselberg, Phys. Rev. A63, 043606 (2001).

21. J. Carlson, S.-Y. Chang, V.R. Pandharipande, and K.E. Schmidt, Phys. Rev. Lett. 91, 050401 (2003).

22. L. Viverit, S. Giorgini, L.P. Pitaevskii, et al., Phys. Rev. A69, 13607 (2004). 